\title{
Il diritto di difesa della vittima nel procedimento di revoca o sostituzione delle misure cautelari personali durante la fase delle indagini preliminari
}

\author{
The Right of Defense of the Victim in the Proceedings \\ of Revocation or Substitution of Precautionary \\ Measures during Preliminary Investigations
}

Filippo Marchetti ${ }^{1}$

Università degli Studi di Pavia - Pavia, Italia filippo.marchetti02@universitadipavia.it https://orcid.org/0000-0003-0596-8929

ABSTRACT: L'autore esamina il procedimento di sostituzione o revoca di una misura cautelare de libertate nell'ottica della verifica del grado di effettività delle garanzie difensive ivi assicurate alla persona offesa. A tal fine, l'analisi abbraccia i profili rappresentati dalla consistenza del compendio informativo a disposizione della vittima in vista dell'instaurazione del contraddittorio, dalla possibilità di presentare elementi utilizzabili per la decisione del giudice e, da ultimo, dalla disponibilità di strumenti per impugnare il provvedimento conclusivo del procedimento.

Parole Chiave: Persona offesa; Misure cautelari; Diritto di difesa.

ABSTRACT: The author examines the processes of substitution or revocation of a precautionary measure to verify the effectiveness of the guarantees ensured therein to the victim. To this end, the analysis embraces the profiles represented by the quality of the dossier at disposal of the victim meant to establish the confrontation with the other parties, by the possibility to submit elements to

1 Dottore di ricerca in Diritto pubblico, giustizia penale e internazionale - Università degli Studi di Pavia. 
the judge, and, finally, by the availability of instruments to challenge the concluding ruling issued by the judge.

KeYwords: Victim; Precautionary measures; Right to defense.

Sommario: Introduzione; 1 . I diritti informativi della persona offesa nel procedimento di sostituzione e revoca delle misure cautelari personali; 2. La consistenza del compendio informativo a disposizione della vittima in vista dell'instaurazione del contraddittorio ex art. 299 c.p.p.; 3. Gli strumenti a disposizione della persona offesa per influire sulla decisione de libertate; 4. Presidi sanzionatori; 5. Modalità di contestazione della violazione delle prerogative partecipative dell'offeso ex art. 299 c.p.p.; Conclusioni; Bibliografia.

\section{INTRODUZIONE}

Fra i settori del processo penale italiano attraversati dal percorso di riscoperta della persona offesa ${ }^{2}$ intrapreso - su impulso proveniente dalla comunità internazionale e in particolare dal diritto europeo ${ }^{3}-$ dal legislatore nazionale, un'attenzione speciale va dedicata al settore delle misure cautelari, sul quale si sono concentrate, a vari livelli, numerose

2 L'ordinamento processuale penale italiano non utilizza, se non in un numero esiguo di casi, il termine vittima (come, invece, accade in sede europea, là dove l'art. 2 Direttiva 2012/29/UE definisce il soggetto in parola come la persona fisica che ha subito un danno, anche fisico, mentale o emotivo, o perdite economiche che sono stati causati direttamente dal reato), bensì quello di persona offesa, intesa quale titolare del bene giuridico offeso dal reato, identificandola, pertanto, col soggetto passivo dell'illecito penale. Come è agevole osservare, le nozioni coincidono solo in parte; poiché, tuttavia, il legislatore nazionale, nell'opera di trasposizione interna delle indicazioni europee, sembra aver ritenuto «sussistere piena equivalenza tra la nomenclatura europea e quella codicistica» (così QUATTROCOLO, Serena. Vittima e processo penale: commistioni di ruoli e di funzioni, Rivista italiana di medicina legale. Milano, n. 2, p. 580, 2018), nel presente lavoro si utilizzeranno quali sinonimi di vittima i termini persona offesa, offeso e soggetto passivo del reato.

3 Al riguardo, BELLUTA, Hervé. Quale ruolo per la vittima nel processo penale italiano?, Revista Brasileira De Direito Processual Penal. Porto Alegre, vol. 5, n. 1, p. 73-92, 2019. 
novelle nel corso degli anni. Nello specifico, due sono i profili che vengono in rilievo: da un lato, quello concernente la morfologia degli strumenti applicabili, ridefinita a partire dal diritto dell'offeso a essere protetto da atti di vittimizzazione ripetuta ${ }^{4}$ da parte dell'imputato ${ }^{5}$; dall'altro, quello attinente alle forme del procedimento de libertate, in relazione al quale si registra l'attribuzione alla vittima di facoltà e diritti di natura tanto informativa, quanto partecipativa.

Quanto al primo aspetto, va brevemente ricordato come il legislatore italiano - nel corso degli anni - abbia ricavato, in seno alla disciplina delle cautele processuali penali, un microsistema ${ }^{6}$ a salvaguardia della persona offesa dal reato. Si tratta, in particolare, di un apparato costituito ora da misure di nuovo conio, ovverosia l'allontanamento dalla casa familiare (art. 282-bis c.p.p.) e il divieto di avvicinamento ai luoghi

4 Per vittimizzazione ripetuta si intende quel fenomeno per il quale la vittima di un reato si trova a subire nuove condotte illecita da parte dello stesso autore o da soggetti a quest'ultimo vicini: al riguardo QUATTROCOLO, Serena. Vulnerabilità e individual assessment: l'evoluzione dei parametri di identificazione, in BARGIS, Marta; BELLUTA, Hervè (a cura di), Vittime di reato e sistema penale. Torino: Giappichelli, 2017, p. 302.

5 Il diritto della vittima alla protezione da condotte offensive dell'imputato trova riconoscimento in numerosi atti sovranazionali. Al riguardo, sia in questa sede consentito richiamare, per quel che riguarda l'ambito euro-unitario, l'art. 18 della Direttiva 2012/29/UE, laddove si prevede l'obbligo per gli Stati membri di proteggere la persona offesa e i suoi familiari da vittimizzazione secondaria e ripetuta, da intimidazioni e ritorsioni, se del caso attraverso procedure istituite a livello nazionale ai fini della protezione fisica dei medesimi soggetti, quali provvedimenti provvisori, ordini di protezione o di non avvicinamento (cfr. punto 52 del Considerando Direttiva 2012/29/UE). Analoghe indicazioni si ricavano, volgendo l'attenzione verso il sistema di protezione dei diritti umani istituito dal Consiglio d'Europa (in particolare, dalla Convenzione di Istanbul, sulla prevenzione e la lotta contro la violenza nei confronti delle donne e la violenza domestica, agli artt. 51 ss.), nonché dalla giurisprudenza della Corte europea dei diritti dell'uomo relativa agli artt. 2, 3 e 8 Cedu. Sul punto, fra gli altri, BONINI, Valentina. Il sistema di protezione della vittima e i suoi riflessi sulla libertà personale. Padova: CEDAM, 2018, p. $53 \mathrm{~s}$.

6 Sottolinea come le novità introdotte dal legislatore in materia finiscano per costituire, all'interno della disciplina generale, una sorta di microsistema, CANZIO, Giovanni. La tutela della vittima nel sistema delle garanzie processuali: le misure cautelari e la testimonianza "vulnerabile", Diritto penale $e$ processo. Milano, n. 10, p. 987, 2010. 
frequentati dalla persona offesa (art. 282-ter c.p.p.) ${ }^{7}$, ora da strumenti tradizionali, se del caso ricalibrati in funzione protettiva - come accade per gli arresti domiciliari di cui all'art. 284 c.p.p., il cui comma 1-bis, introdotto dal D.L. $1^{\circ}$ luglio 2013, n. 78, conv. con mod. 1.9 agosto 2013, n. 94, impone al giudice di individuare il locus custodiae «in modo da assicurare comunque le prioritarie esigenze di tutela della persona offesa dal reato»-.

È, tuttavia, il secondo profilo, quello legato all'inserimento della vittima quale nuovo contraddittore nel contesto in esame, «per natura estraneo al coinvolgimento di altri soggetti privati» ${ }^{8}$ oltre all'imputato, a destare maggiore interesse, in quanto capace di fornire un piano di indagine privilegiato per saggiare la capacità dell'ordinamento processuale di far fronte alle nuove esigenze di cui è portatore il soggetto in parola. In questa direzione si colloca, infatti, la creazione - ad opera del D.L. 14 agosto 2013, n. 93, conv. con mod. 1.15 ottobre 2013, n. 119 - del meccanismo partecipativo a disposizione della persona offesa in seno al segmento procedimentale dedicato alla sostituzione o alla revoca delle misure cautelari personali di cui all'art. 299, commi 3 e 4-bis c.p.p. ${ }^{9}$, che si prefigge lo scopo di assicurare al soggetto in parola la concreta possibilità di influire sulle scelte del giudice in materia di adeguamento in melius del regime cautelare in atto.

7 Al riguardo, BRONZO, Pasquale. Le "nuove" misure prescrittive, in AA. VV., Il pluralismo delle misure cautelari personali. Padova: CEDAM, 2017, p. 55-95; CARACENI, Lina. Misure cautelari pro victima e diritti di libertà dell'accusato: a proposito di una convivenza faticosa, Archivio della nuova procedura penale. Piacenza, n. 3, p. 254-270, 2017; NEGRI, Daniele. Le misure cautelari a tutela della vittima: dietro il paradigma flessibile, il rischio di un'incontrollata prevenzione, Giurisprudenza italiana, Milano, n. 2, c. 467-474, 2012; ZACCHÈ, Francesco. Criterio di necessità e misure cautelari. Milano: Giuffrè, 2018, p. 33 s.

8 BONTEMPELLI, Manfredi. Novità nelle procedure di revoca e sostituzione, in DIDDI, Alessandro; GERACI, Rosa Maria (a cura di), Misure cautelari ad personam in un triennio di riforme. Torino: Giappichelli, 2015, p. 144.

9 Sul punto, v. infra § 1. Sul contraddittorio cautelare, in generale, v. CRISTIANI, Antonio. Aspetti problematici del contraddittorio nel riesame dei provvedimenti restrittivi della libertà personale, Rivista italiana di diritto e procedura penale. Milano, n. 3, p. 967, 1984; MOLARI, Alfredo. Introduzione, in AA. VV., Il contraddittorio tra Costituzione e legge ordinaria. Milano: Giuffrè, 2002, p. 137 s. 
Orbene, il nuovo assetto delle prerogative della vittima nell'incidente de libertate non ha mancato di suscitare, a vari livelli, critiche ed obiezioni volte, per la maggior parte, a sottolinearne i riflessi pregiudizievoli sui diritti dell'imputato ${ }^{10}$. Mutando parzialmente prospettiva, in questa sede si intende, invece, riguardare l'interpello cautelare dell'offeso ex art. 299 c.p.p., instaurato durante la fase delle indagini preliminari, nell'ottica della verifica circa la sua reale capacità di soddisfare l'esigenza che ha spinto il legislatore alla sua forgiatura: si cercherà, in altri termini, di rispondere al quesito se esso costituisca, o meno, un'effettiva e concreta chance per la persona offesa di portare il proprio contributo alla decisione del giudice. Procedendo in questo senso, dopo una breve panoramica dei presupposti dell'istituto, si prenderanno in esame i profili che, tenuto conto delle specificità del rito cautelare, paiono gli indici più significativi per l'indagine che ci si ripropone di compiere: la conoscibilità, da parte della vittima, degli elementi probatori ed argomentativi che costituiscono il materiale utilizzabile per la decisione del giudice; la disponibilità in capo all'offeso di strumenti per influire, su basi di parità rispetto agli altri contendenti, sulla pronuncia giudiziale; $\mathrm{e}$, infine, i rimedi a disposizione del medesimo soggetto avverso provvedimenti decisori pronunciati in spregio alle proprie prerogative partecipative, o senza tener conto delle proprie ragioni.

$10 \mathrm{~V}$., in tal senso, il giudizio fortemente critico di AMODIO, Ennio. Relazione introduttiva, in AA. VV., I nuovi orizzonti della giustizia penale europea. Milano: Giuffrè, 2015, p. 27, per il quale il «contraddittorio sulla libertà personale, introdotto con la l. n. 119/2013 che ha convertito il d.l. n. 93/2013, trasforma l'offeso nel titolare di una accusa privata che può rendersi interprete di istanze di pura vendetta». Analogamente, ma in termini più sfumati, CATALANO, Elena Maria. La tutela della vittima nella direttiva 2012/29/UE e nella giurisprudenza delle corti europee, Rivista italiana di diritto e procedura penale. Milano, n. 4, p. 1804, 2014; DIDDI, Alessandro. Chiaroscuri nella nuova disciplina sulla violenza di genere, Processo penale e giustizia. Torino, n. 2, p. 100, 2014; TODARO, Guido. Il sistema italiano di tutela della vittima: analisi e prospettive, in LUPARIA, Luca (a cura di), Lo statuto europeo delle vittime di reato. Padova: CEDAM, 2015, p. 108. 


\section{I DIRITTI INFORMATIVI DELLA PERSONA OFFESA NEL PROCEDIMENTO DI SOSTITUZIONE E REVOCA DELLE MISURE CAUTELARI PERSONALI.}

Le prerogative assegnate alla vittima nella dinamica cautelare si compongono di diritti informativi funzionali ora a mettere al corrente il soggetto de quo del mutamento dello status libertatis dell'imputato ${ }^{11}$, ora a consentirgli di partecipare attivamente al procedimento.

Quanto ai primi, si prevede che la persona offesa sia informata dell'avvenuta adozione di uno tra gli ordini di protezione disciplinati dagli artt. 282-bis e 282-ter c.p.p. (art. 282-quater c.p.p.). Accanto a questo iniziale bagaglio informativo, l'art. 299, comma 2-bis c.p.p., così come interpolato dal D.L. 14 agosto 2013, n. 93, conv. con mod. 1.15 ottobre 2013, n. 119, dispone che i provvedimenti di revoca, sostituzione o applicazione con modalità meno gravose di una delle misure di cui agli artt. 282-bis, 282-ter, 283, 284, 285, 286 c.p.p., applicate in procedimenti commessi con violenza alla persona, siano immediatamente comunicati, a cura della polizia giudiziaria, ai servizi socio-assistenziali, alla persona offesa e al suo difensore, se nominato. Sempre nel contesto di procedimenti per reati violenti, alla vittima che ne abbia fatto richiesta devono inoltre essere comunicati, con l'ausilio della polizia giudiziaria, i provvedimenti di scarcerazione e di cessazione di una misura di sicurezza detentiva, nonché l'evasione dell'imputato detenuto o del condannato, e la sottrazione dell'internato all'esecuzione della misura di sicurezza detentiva, sempre che non risulti un pericolo concreto di danno per l'autore del reato (art. 90-ter, comma 1 c.p.p., inserito dal D. Lgs. 15 dicembre 2015, n. 212) ${ }^{12}$.

11 Anche in questo caso l'azione del legislatore nazionale si è mossa lungo i binari tracciati dalla normativa internazionale, e in particolare dall'art. 6, parr. 5 e 6 della Direttiva 2012/29/UE, il quale obbliga gli Stati a prevedere che la persona offesa sia informata, previa richiesta e senza indebito ritardo, della scarcerazione o dell'evasione dell'imputato sottoposto a custodia quantomeno nelle eventualità in cui il riacquisto dello status libertatis da parte di quest'ultimo determini un pericolo per la vittima o per i suoi familiari. Analoghe indicazioni provengono anche dall'art. 56, par. 1 , lett. $b$ della Convenzione di Istanbul.

12 Recentemente, la 1. 19 luglio 2019, n. 69 ha inserito, nel corpo dell'art. 90-ter c.p.p., il comma 1-bis, in base al quale le comunicazioni previste dal comma 1 della medesima disposizione sono sempre effettuate, senza che sia necessaria una richiesta in tal senso, qualora si proceda per uno dei delitti previsti dagli 
Per quanto riguarda, invece, le prerogative funzionali all'instaurazione del contraddittorio cautelare con la vittima, l'attenzione del legislatore è tornata sul procedimento di immutazione del regime cautelare, coniando un inedito interpello della persona offesa da reati di natura violenta, che presenta tratti alquanto peculiari. In questo senso, l'art. 299, comma 3 c.p.p. stabilisce, infatti, che, durante le indagini preliminari, la parte che chieda la sostituzione o la revoca di una delle misure cautelari coercitive di cui agli artt. 282-bis, 282-ter, 283, 284, 285, 286 c.p.p. debba contestualmente notificare, a pena di inammissibilità, la relativa istanza al difensore della vittima o, in mancanza di quest'ultimo, alla persona offesa salvo che, in quest'ultimo caso, essa non abbia provveduto a dichiarare o a eleggere domicilio ${ }^{13}$. In questo modo, l'offeso - o il proprio difensore - è messo nelle condizioni, nei due giorni successivi alla notifica, di presentare memorie ${ }^{14}$ al giudice, il quale deve attendere, prima di poter decidere, il decorso del predetto termine ${ }^{15}$.

Come è agevole osservare, il meccanismo volto a coinvolgere l'offeso nelle dinamiche custodiali non rappresenta un istituto a carattere

artt. 572, 609-bis, 609-ter, 609-quater, 609-quinquies, 609-octies e 612-bis c.p., nonché dagli artt. 582 e 583-quinquies c.p., nelle ipotesi aggravate ai sensi degli artt. 576, comma 1, nn. 2, 5 e 5.1, e 577, comma 1, n. 1 e 2 c.p.

13 L'articolo in commento esclude l'obbligo di notifica qualora l'istanza sia formulata nel contesto dell'adempimento di cui all'art. 294 c.p.p. Si tratta del c.d. interrogatorio di garanzia che il giudice competente deve effettuare, fino alla dichiarazione di apertura del dibattimento, entro cinque giorni dall'esecuzione dell'ordinanza applicativa della custodia cautelare; termine, quest'ultimo, esteso a dieci giorni qualora siano adottate misure diverse, sia coercitive che interdittive. La ratio dell'eccezione in parola va individuata, secondo l'opinione dottrinale maggioritaria, nell'intenzione del legislatore di mantenere inalterate la celerità e la funzione eminentemente difensiva dell'istituto di cui all'art. 294 c.p.p. In questi termini, VALENTINI, Elena. Il ruolo della persona offesa nella procedura di revoca e sostituzione delle misure cautelari personali, in CURI, Francesca (a cura di), Ordine pubblico e sicurezza nel governo della città. Bologna: BUP, 2016, p. 232.

14 Sugli strumenti a disposizione della persona offesa v. infra, § 3.

15 Nella fase successiva all'esercizio dell'azione penale il coinvolgimento della persona offesa del procedimento di sostituzione o revoca di una misura cautelare coercitiva trova realizzazione, ex art. 299, comma 4-bis c.p.p., attraverso un meccanismo che ricalca, con qualche minima variazione, quello delineato nel testo. 
generale, essendo il suo innesco subordinato al ricorrere di taluni presupposti che ne limitano significativamente il campo di applicazione.

Innanzitutto, il contraddittorio cautelare con la vittima risulta confinato all'interno dei soli procedimenti per delitti commessi con violenza alla persona. Formula, quest'ultima, non particolarmente perspicua, e che, come tale, ha reso necessario un intenso lavorio ermeneutico da parte degli interpreti al fine di precisarne la portata in termini di minor vaghezza, senza che, peraltro, si sia arrivati ad una soluzione realmente soddisfacente. Al riguardo va, infatti, ricordato come la dottrina abbia proposto diverse interpretazioni, infine coagulatesi intorno a due orientamenti principali. Secondo una prima impostazione, facente perno su di un'interpretazione strettamente ancorata alla littera legis, la disposizione in commento sarebbe evocativa dei soli reati commessi con la violenza fisica ${ }^{16}$. Altri, invece, privilegiando un'ermeneutica estensiva, ritengono che la formula prescelta dal legislatore dovrebbe ritenersi comprensiva anche della violenza morale e psicologica ${ }^{17}$.

Non meno variegato si presenta il panorama giurisprudenziale. Accanto, infatti, a pronunce che hanno fatto proprio l'orientamento dottrinale da ultimo ricordato ${ }^{18}$, si registra un filone giurisprudenziale di recente emersione, in base al quale la formula «delitti commessi con violenza alla persona» alluderebbe a reati - commessi con violenza tanto fisica, quanto morale - cui si è soliti associare una particolare vulnerabilità

16 Così VALENTINI, Elena. Il ruolo della persona offesa nella procedura di revoca e sostituzione, cit., p. 236.

17 In tal senso, ZACCHÈ, Francesco. Le cautele fra prerogative dell'imputato e tutela della vittima di reati violenti, Rivista italiana di diritto e procedura penale. Milano, n. 2, p. 673, 2015.

18 In tal senso, Cass. Pen., sez. III, 8 febbraio 2017, n. 5832, D., Giurisprudenza italiana. Milano, n. 2, p. 424 s, 2019. Alcune delle pronunce appartenenti al filone giurisprudenziale in commento richiedono, a guisa di presupposto ulteriore rispetto a quelli indicati nel testo, che l'agito violento si inscriva in un pregresso rapporto fra vittima e imputato, con l'effetto di escludere dal campo di applicazione dell'istituto in parola i procedimenti per reati in cui la violenza sia del tutto occasionale: così Cass. Pen., sez. II, 19 settembre 2018, n. 50064, R., Diritto \& giustizia. Milano, 9 gennaio 2019. Per una critica a siffatta impostazione, v. RUGGIERO, Rosa Anna. La tutela processuale della violenza di genere, Cassazione penale. Milano: Giuffrè, n. 6, p. 2353, 2014. 
della vittima alla vittimizzazione ripetuta (ovverosia illeciti riconducibili alla tratta di esseri umani, al terrorismo, alla criminalità organizzata, alla violenza e allo sfruttamento sessuale, alla criminalità d'odio, o, ancora, alla violenza di genere e quella nelle relazioni strette) ${ }^{19}$.

L'istituto in commento è, poi, destinato ad operare solo allorché l'istanza cautelare presentata dall'imputato o dal pubblico ministero sia volta alla revoca ovvero alla sostituzione della misura originariamente applicata ${ }^{20}$. Non è, invece, prescritto il coinvolgimento obbligatorio dell'offeso nell'ipotesi in cui il giudice proceda ex officio ad adeguare in melius il regime cautelare in atto ${ }^{21}$; né alcuna comunicazione deve

19 Così Cass. Pen., sez. II, 23 aprile 2020, n. 12800, C., Processo penale e giustizia. Torino, n. 6, p. 1433 s., 2020. Al riguardo, criticamente, ROMANELLI, Bartolomeo. La persona offesa vulnerabile nel procedimento penale. Milano: EDUCatt, 2020, p. 142 s.

20 Sul punto ci si potrebbe domandare se il riferimento, all'interno dell'art. 299, comma 3 c.p.p., alla sostituzione possa indurre a ritenere necessario il coinvolgimento della persona offesa anche nell'ipotesi di aggravamento della misura cautelare. Al riguardo, parte della dottrina ritiene di rispondere negativamente al quesito poc'anzi posto, facendo perno sulla disparità di trattamento che la risposta positiva potrebbe produrre fra persona offesa e imputato, il quale, nell'ipotesi di sostituzione in peius, non gode del diritto a rendere interrogatorio. Così VALENTINI, Elena. Il ruolo della persona offesa nella procedura di revoca e sostituzione, cit., p. 230.

Alle considerazioni sopra riportate si potrebbe aggiungere che la stessa collocazione dei diritti informativi e partecipativi della persona offesa all'interno dei commi 3 e 4-bis dell'art. 299 c.p.p., che si occupano della revoca e della modifica in melius, sembra corroborare la tesi dell'esclusione della richiesta in malam partem dal campo di applicazione del meccanismo di coinvolgimento dell'offeso.

Ancora aperto rimane, invece, il quesito circa la doverosità, o meno, della notificazione alla vittima della domanda che solleciti, non la sostituzione della cautela originariamente applicata, bensì la semplice immutazione in bonam partem delle sue modalità esecutive. Sul punto sia consentito rinviare, anche per gli opportuni riferimenti bibliografici, a MARCHETTI, Filippo. L'intervento dell'offeso nel procedimento di revoca e sostituzione di una misura cautelare personale nuovamente sotto la lente della Suprema Corte, Diritto penale contemporaneo. Milano, n. 6, p. 121-133, 2019.

21 Ai sensi dell'art. 299, comma 3 c.p.p., il giudice può procedere ex officio a revocare o a sostituire in melius la misura cautelare in atto al momento di assumere l'interrogatorio della persona in stato di custodia cautelare; nell'ipotesi in cui sia richiesto della proroga del termine delle indagini preliminari o 
essere fornita alla vittima nel caso in cui l'istanza di parte sia diretta a far valere taluna delle cause estintive delle misure di cui agli artt. 300 e ss. c.p.p. ${ }^{22}$.

Infine, l'art. 299, comma 3 e 4-bis c.p.p., nella parte in cui impone la notifica dell'istanza «presso il difensore della persona offesa o, in mancanza di questo, alla persona offesa, salvo che in quest'ultimo caso essa non abbia provveduto a dichiarare o eleggere domicilio», sembra porre in capo alla vittima che voglia partecipare all'incidente cautelare l'onere di nominare un patrono o - in alternativa - di dichiarare o di eleggere un domicilio per le notificazioni ${ }^{23}$.

dell'assunzione dell'incidente probatorio; ovvero quando procede all'udienza preliminare $o$ al giudizio.

Si tratta, in particolare, di ipotesi in cui l'effetto estintivo della misura non dipende da una rivalutazione dei presupposti cautelari da parte del giudice, bensì dal mero accertamento del verificarsi di taluni presupposti legati alla prosecuzione del procedimento, quali: la pronuncia di talune sentenze (art. 300 c.p.p.); il superamento del limite di durata della misura adottata per esigenze probatorie (art. 301 c.p.p.); il mancato compimento dell'interrogatorio di cui all'art. 294 c.p.p. nei termini prescritti (art. 302 c.p.p.); e, infine, il superamento dei termini massimi di custodia (artt. 303 e 308 c.p.p.).

Nel senso indicato nel testo, Cass. Pen., sez. V, 11 dicembre 2018, n. 5717, C., Rv. 275137.

In tal senso, LOMBARDI, Filippo. Tutela della vittima nella vicenda cautelare e obbligo di notificare l'istanza di revoca (o modifica) della misura, Processo penale e giustizia. Milano, n. 6, p. 1527, 2020; VALENTINI, Elena. Il ruolo della persona offesa nella procedura di revoca e sostituzione, cit., p. 231-232.

$\mathrm{Al}$ riguardo va, tuttavia, detto che la formula poco adamantina impiegata dal legislatore ha favorito la formazione, all'interno della giurisprudenza di legittimità, di due orientamenti contrapposti. Mentre, infatti, alcune sentenze hanno fatto propria la tesi dottrinale indicata nel testo, altre pronunce hanno, invece, ritenuto che l'art. 299 c.p.p. non imponga alla vittima alcun onere, di talché la notificazione dell'istanza di sostituzione e revoca le sarebbe dovuta anche nel caso in cui la stessa non abbia nominato un difensore, eletto o dichiarato domicilio. Da ultimo è accaduto che la I Sezione della Corte di cassazione, rilevando l'esistenza dell'accennata contrapposizione interpretativa, ha rimesso la questione alle Sezioni Unite affinché appiani il contrasto ermeneutico: v. Cass. Pen., sez. I, ord., 4 maggio 2021, n. 22444, Gallo, consultabile al seguente indirizzo https://www.cortedicassazione.it/corte-di-cassazione/ it/p_questioni_pendenti.page. 


\section{LA CONSISTENZA DEL COMPENDIO INFORMATIVO A DISPOSIZIONE DELLA VITTIMA IN VISTA DELL'INSTAURAZIONE DEL CONTRADDITTORIO EX ART. 299 C.P.P.}

Proseguendo lungo la linea di sviluppo tracciata in premessa, occorre focalizzare l'attenzione sulle indagini preliminari, fase in cui, per un verso, è statisticamente più frequente che si pongano questioni de libertate e, per l'altro, l'esercizio delle prerogative difensive dei soggetti privati coinvolti è reso maggiormente disagevole a cagione della segretezza che connota gli atti ivi compiuti dal pubblico ministero ${ }^{24}$. Al riguardo, va in via preliminare ricordato come il procedimento di sostituzione o revoca di una misura cautelare coercitiva ivi attivato si svolga de plano, ovverosia senza che a seguito del relativo atto introduttivo segua la fissazione di un'udienza ad hoc. In tale contesto, quindi, la realizzazione del contraddittorio - di norma, meramente cartolare - avviene attraverso «battute asincrone», con conseguente necessità, perché la contrapposizione dialettica possa dirsi realmente efficace, che «ognuno dei contendenti conosca non soltanto l'oggetto della contesa, ma anche la posizione e gli argomenti dell'altro», giacché «due [o più] pareri sull'argomento non costituiscono contraddittorio» ${ }^{25}$.

A questo riguardo è, peraltro, noto che il rito de libertate, quantomeno nella fase preliminare, risulta imperniato su di un modello gnoseologico caratterizzato da una spiccata frammentarietà, giacché ciascuno dei suoi tradizionali protagonisti - il giudice, il pubblico ministero e l'accusato - ha a disposizione un differente patrimonio conoscitivo ${ }^{26}$. Focalizzando l'attenzione sul segmento che qui più interessa, ovverosia

24 Sul diritto di difesa dell'accusato nel procedimento de libertate v., anche per gli opportuni riferimenti bibliografici, GIULIANI, Livia. Autodifesa e difesa tecnica nei procedimenti de libertate. Padova: CEDAM, 2012, passim.

25 La presente citazione, insieme con le due immediatamente precedenti, è tratta da GIOSTRA, Glauco. voce Contraddittorio (principio del) II) Diritto processuale penale, in Enciclopedia giuridica Treccani. VIII. Roma: Treccani, 2001, p. 1. Analogamente, con riferimento anche al procedimento cautelare de libertate, UBERTIS, Giulio. Sistema di procedura penale. IV ed. vol. I. Milano: Giuffrè, 2017, p. 181.

26 Sul punto, DI CHIARA, Giuseppe. Linee di sistema della funzione giudiziale preliminare, Rivista di diritto processuale. Milano, n. 1, p. 243, 2003. 
quello dedicato alla revoca o alla sostituzione in melius delle misure cautelari personali, si osserva come in tale contesto ciascuno dei soggetti poc'anzi ricordati possieda - accanto ad un compendio di conoscenze in comune, rappresentato dagli elementi posti alla base della misura cautelare originariamente applicata ${ }^{27}$ - informazioni diversificate. La parte pubblica, infatti, ha piena contezza anche «del dossier dell'accusa, coperto da segreto investigativo e, pertanto, nella sua interezza inaccessibile a chiunque in pendenza di fase ${ }^{28}$; l'indagato, invece, non riceve - prima della decisione del giudicante - alcuna comunicazione circa le eventuali

27 Per quanto riguarda l'indagato e il suo difensore, la discovery degli elementi posti dal pubblico ministero a corredo della domanda di applicazione di una misura cautelare personale ex art. 291 c.p.p., e utilizzati dal giudice per la decisione, avviene a norma dell'art. 293, comma 3 c.p.p., in base al quale l'ordinanza applicativa della cautela, dopo la sua esecuzione o la sua notificazione, è depositata nella cancelleria del giudice che l'ha emessa insieme con la richiesta del pubblico ministero e agli atti presentati con la stessa. Si tratta, come autorevolmente segnalato in dottrina, di un adempimento funzionale ad un effettivo esercizio del diritto di difesa nel procedimento de libertate, in quanto «destinat[o] a rafforzare le garanzie dell'imputato non solo nella prospettiva della procedura di riesame di fronte al tribunale della libertà, ma anche, prima ancora, in ogni momento successivo al deposito»: così GREVI, Vittorio. Alla ricerca di un processo penale «giusto». Milano: Giuffrè, 2000, p. 56. A parte ciò, ai sensi dell'art. 366 c.p.p., il difensore dell'indagato ha, sin da subito, diritto a esaminare ed estrarre copia dei verbali degli atti del pubblico ministero e della polizia giudiziaria al cui compimento abbia diritto di assistere.

L'art. 299 c.p.p. non prevede espressamente che il giudice, per decidere su di un'istanza di sostituzione o revoca della cautela in atto, abbia accesso agli atti presentati dal pubblico ministero a supporto dell'iniziativa cautelare ex art. 291 c.p.p., e posti a fondamento della misura precedentemente applicata. Cionondimeno, si ritiene che gli elementi in parola debbano essere trasmessi al giudicante, eventualmente anche su sua richiesta, dalla parte pubblica. V., al riguardo, LEONASI, Raffaele; FERRANTI, Donatella. Il contraddittorio nell'applicazione, revoca e modifica delle misure cautelari, Cassazione penale. Milano, n. 8-9, p. 1653-1654, 1990; MANZIONE, Domenico. Commento all'art. 299 c.p.p., in CHIAVARIO, Mario (a cura di), Commento al codice di procedura penale. Agg. III. Torino: UTET, 1998, p. 303; MARZADURI, Enrico. voce Misure cautelari personali (principi generali e disciplina). In Digesto delle discipline penalistiche. Vol. VIII. Torino: UTET, 1994, p. 88-89.

DI CHIARA, Giuseppe. Linee di sistema della funzione giudiziale preliminare, cit., p. 244. 
produzioni e deduzioni del pubblico ministero ${ }^{29}$; mentre il giudice dispone «di una selezione di atti offertigli dalla parte istante, eventualmente arricchiti dalle conoscenze acquisite in occasione di precedenti decisioni incidentali richiestegli dalle parti» ${ }^{30}$, dai risultati delle investigazioni difensive presentatigli dai difensori ex art. 391-octies, comma 2 c.p.p. ${ }^{31}$, e - qualora la richiesta ex art. 299 c.p.p. sia presentata dall'accusato, ovvero il giudice proceda ex officio ad adeguare in melius il regime cautelare - dal parere del magistrato inquirente formulato ex art. 299, comma 3-bis c.p.p. ${ }^{32}$.

Tale assetto normativo, apparso - da tempo - non del tutto soddisfacente nell'ottica della garanzia per il bene supremo della libertà personale dell'accusato ${ }^{33}$, fa registrare, se riguardato dalla prospettiva della tutela delle prerogative defensionali dell'offeso, un ugualmente

29 Al riguardo va, infatti, ricordato che parte della giurisprudenza ritiene, facendo ricorso all'analogia con quanto disposto dall'art. 293, comma 3 c.p.p., che il giudice, laddove rigetti un'istanza di revoca o di modifica in melius del regime cautelare, abbia l'obbligo di depositare, dopo aver deciso, il parere formulato dal pubblico ministero ai sensi dell'art. 299, commi 3-bis e 4-bis c.p.p., nonché gli ulteriori atti dal medesimo eventualmente prodotti. In tal senso, Cass. Pen., sez. II, 20 dicembre 2000, n. 2063, Iussi, Rv. 217830; Cass. Pen., sez. VI, 10 marzo 1997, n. 976, Sassanelli, Rv. 208113.

30 DI CHIARA, Giuseppe. Linee di sistema della funzione giudiziale preliminare, cit., p. 244.

31 Per quanto riguarda l'ostensione dei risultati delle investigazioni difensive depositati ex art. 391-octies, comma 2 c.p.p. nei confronti degli altri contendenti processuali, l'art. 391-octies, comma 3 c.p.p. dispone che il pubblico ministero possa prenderne visione ed estrarne copia solo prima che venga adottata una decisione del giudice sollecitata dalle altre parti o con il loro intervento. Non è, invece, prevista alcuna discovery a favore delle parti private diverse dall'imputato o della persona offesa. Al riguardo, LORENZETTO, Elisa. Il diritto di difendersi indagando nel sistema processuale penale. Napoli: ESI, 2013, p. $676 \mathrm{~s}$.

32 L'art. 299, comma 3-bis c.p.p. dispone, al riguardo, che il giudice, prima di provvedere in ordine alla revoca o alla sostituzione delle misure coercitive e interdittive, di ufficio o su richiesta dell'imputato, deve sentire il pubblico ministero. Se questi, però, non esprime, nei due giorni successivi, il proprio parere, il giudicante può comunque procedere.

33 Cfr. FERRAIOLI, Marzia. Il ruolo di «garante» del giudice per le indagini preliminari. IV ed. Padova: CEDAM, 2014, p. 183. Nonché RUGGERI, Stefano. Equality of arms, impartiality of the judiciary and the role of the parties in the pre-trial inquiry: the perspective of Italian criminal justice, Revista Brasileira De Direito Processual Penal. Porto Alegre, vol. 4, n. 2, p. 559-603, 2018. 
significativo deficit di tutela, che pare addebitabile, in ultima analisi, alla scarsa cura prestata al tema da parte del legislatore.

Per convincersene basti soffermare l'attenzione sulla portata dell'orizzonte conoscitivo che si schiude dinanzi al soggetto passivo del reato al momento della presentazione di un'istanza ex art. 299 c.p.p. La disposizione poc'anzi citata dispone in proposito che alla vittima debba essere notificata la richiesta de libertate ivi contemplata, ovverosia il solo atto con il quale l'istante chieda al giudice la rimozione o una variazione in melius del regime cautelare, insieme con le argomentazioni, in fatto e in diritto, a supporto dello stesso. Non rientrano, invece, nel campo di applicazione della disposizione tutte le produzioni o allegazioni che, pur accedendo alla richiesta in senso stretto, possano essere logicamente e materialmente separate da quest'ultima: ci si riferisce, in particolare, a eventuali documenti ovvero ai risultati delle investigazioni difensive ${ }^{34}$. Né, laddove la richiesta di sostituzione o revoca sia formulata dall'indagato, è prevista alcuna forma di ostensione nei confronti dell'offeso del parere eventualmente espresso dal pubblico ministero ex art. 299, comma 3-bis c.p.p. ${ }^{35}$.

Oltre alle lacune conoscitive poc'anzi evidenziate, vi sono altre ragioni che contribuiscono a penalizzare la posizione del soggetto passivo del reato nel procedimento de quo rispetto a quella degli altri contendenti.

34 Oltre che dall'argomento testuale, l'interpretazione ricordata nel testo pare l'unica praticabile anche facendo ricorso a considerazioni di tipo sistematico. In questo senso, si pensi, solo per fare qualche esempio, alla richiesta di rimessione del processo che, ex art. 46, comma 1 c.p.p., deve essere depositata, con i documenti che vi si riferiscono, nella cancelleria del giudice ed è notificata entro sette giorni a cura del richiedente alle altre parti. Orbene, anche in questo caso si ritiene che l'istante sia onerato della notifica della sola domanda, non già anche degli ulteriori elementi depositati: in tal senso, GIULIANI, Livia. Rimessione del processo e valori costituzionali. Torino: Giappichelli, 2002, p. 242.

Analoghe considerazioni valgono anche per l'ipotesi contemplata dall'art. 395 c.p.p., che onera la parte che chieda l'incidente probatorio, per un verso, di depositare, nella cancelleria del giudice per le indagini preliminari, la richiesta insieme con cose o documenti e, per l'altro, di notificare agli altri interessati la sola istanza: in tal senso DI CHIARA, Giuseppe. voce Incidente probatorio, in Enciclopedia del diritto. Agg. VI. Milano: Giuffrè, 2002, p. 558.

Cfr. RUSSO, Carmine. Femminicidio. Milano: Giuffrè, 2013, p. 34. 
A questo riguardo va, infatti, ricordato che, per quanto non manchino occasioni nelle quali, nell'arco della fase investigativa, si aprono spazi di discovery degli atti in favore della difesa della vittima - si pensi, solo per fare un paio di esempi, al compimento di accertamenti tecnici non ripetibili (art. 360 c.p.p.), ovvero all'assunzione dell'incidente probatorio (art. 392 c.p.p.), in relazione ai quali il difensore del soggetto in parola gode del diritto tanto alla partecipazione, quanto all'esame e all'estrazione di copia dei relativi verbali (artt. 366 e 401, comma 8 c.p.p.) ${ }^{36}-$, nessuna norma prevede che l'offeso sia informato del deposito del fascicolo cautelare, né che possa avervi accesso. Si tratta, come noto, del compendio di atti presentati dal pubblico ministero in occasione della formulazione di una richiesta di applicazione di una misura cautelare personale ai sensi dell'art. 291 c.p.p..$^{37}$, che, oltre ad essere oggetto di disclosure nei confronti dell'accusato, viene messo a disposizione del giudice, non solo per la decisione sull'adozione della cautela, ma anche in vista delle successive, eventuali pronunce in materia de libertate durante la fase preliminare ${ }^{38}$. Di tutti questi atti, dunque, la vittima, al momento della presentazione di un'istanza ex art. 299 c.p.p., potrebbe avere una conoscenza solo parziale, e precisamente limitata agli elementi già oggetto di ostensione nei suoi confronti durante il corso delle indagini ${ }^{39}$.

36 Sul punto, QUAGLIERINI, Corrado, Le parti privati diverse dall'imputato e l'offeso dal reato, in UBERTIS, Giulio; VOENA Giovanni Paolo (diretto da), Trattato di procedura penale. vol. VIII. Milano: Giuffrè, 2003, p. 164 s.; PAULESU, Pier Paolo, Vittima del reato e processo penale: uno sguardo d'insieme (informazioni, diritti, tutele), in BARGIS, Marta; BELLUTA, Hervè (a cura di), Vittime di reato e sistema penale. Torino: Giappichelli, 2017, p. 150.

Una completa discovery degli atti di indagine si realizza, a favore della persona offesa, solo nella fase conclusiva delle indagini preliminari, sia che il pubblico ministero si orienti verso la richiesta di archiviazione (art. 408, comma 3 c.p.p.), sia nell'eventualità in cui la parte pubblica eserciti l'azione penale contro l'accusato (artt. 419 c.p.p. e 131 disp. att. c.p.p.). Al riguardo, PANSINI, Carla. Contributo dell'offeso e snodi procedimentali. Padova: CEDAM, 2004, p. 30 s.

37 Al riguardo, funditus, VALENTINI, Elena. La domanda cautelare nel sistema delle cautele personali. Bologna: BUP, 2012, p. 117 s. V. retro, nota n. 27.

39 Al riguardo va, inoltre, ricordato che, salvo il caso in cui lo strumento cautelare originariamente adottato sia l'allontanamento dalla casa familiare - o il divieto di avvicinamento alla persona offesa - il cui provvedimento applicativo 
Orbene, si potrebbe, prima facie, pensare di colmare la segnalata lacuna informativa per via ermeneutica, argomentando a partire dall'art. 116 c.p.p., che, come è noto, consente - in via generale - a chiunque vi abbia interesse di chiedere il rilascio a proprie spese di copie, estratti o certificati di singoli atti. In effetti, l'articolo poc'anzi richiamato è parso, secondo un'impostazione dottrinale, funzionale a consentire al difensore dell'offeso l'accesso agli atti, «che non sono coperti da segreto, in quanto noti alla persona sottoposta alle indagini ${ }^{40}$. Sennonché, melius re perpensa, esso si rivela uno strumento inadeguato ai nostri fini per un duplice ordine di ragioni. In primo luogo, la disposizione in commento, nella misura in cui demanda, a seconda dei casi, al pubblico ministero o al giudice un vaglio discrezionale in merito all'accoglimento, o meno, della relativa istanza, non sembra conferire al difensore della vittima, quantomeno nell'ipotesi in commento, un diritto incondizionato ad ottenere copia degli atti ${ }^{41}$; secondariamente, l'assenza di alcun termine per il rilascio sottende il rischio che la reale disponibilità degli elementi richiesti non giunga in tempo utile per l'esercizio delle facoltà attribuite in seno al procedimento di cui all'art. 299 c.p.p. che, invece, risulta scandito secondo rigide tempistiche.

Ragioni analoghe inducono a scartare anche l'opzione interpretativa che facesse perno sull'art. 335, comma 3-ter c.p.p., in base al quale, senza pregiudizio del segreto investigativo, decorsi sei mesi dalla data di presentazione della denuncia, ovvero della querela, la persona offesa dal reato può chiedere di essere informata dall'autorità che ha in carico il procedimento circa lo stato del medesimo. A parte ogni considerazione in merito all'impossibilità di valersi del mezzo de quo quando l'istanza di sostituzione o revoca di una misura cautelare fosse formulata prima

deve, come si è visto, essere comunicato alla vittima, quest'ultima potrebbe financo essere all'oscuro dell'ordinanza de libertate adottata dal giudice ex art. 292 c.p.p., e dunque anche delle argomentazioni che sorreggono la misura di cui si chiede la sostituzione o la revoca.

40 Così NAPPI, Aniello. Guida al codice di procedura penale. X ed. Milano: Giuffrè, 2007, p. 94.

41 Sul punto, in generale, DE AMICIS, Gaetano. Appunti per una ricostruzione sistematica del diritto alla consultazione degli atti processuali, Cassazione penale. Milano, n. 3, p. 978, 1996. 
dello spirare del termine dilatorio di sei mesi dalla presentazione della denuncia o della querela, una soluzione ermeneutica di tal fatta dovrebbe comunque confrontarsi con il tenore letterale della disposizione che, pur nell'estrema genericità che la contraddistingue ${ }^{42}$, sembrerebbe alludere a informazioni relative all'avanzamento delle indagini e alle determinazioni del pubblico ministero, mentre difficilmente potrebbe contemplare un vero e proprio diritto all'ostensione di specifici atti di indagine in aggiunta alle ipotesi di discovery contemplate nel sistema ${ }^{43}$.

\section{Gli strumenti a disposizione della persona offesa Per INFLUIRE SULLA DECISIONE DE LIBERTATE.}

Una volta conclusa l'analisi circa la consistenza delle informazioni a disposizione dell'offeso in vista della costituzione del contraddittorio cautelare ex art. 299 c.p.p., occorre soffermare l'attenzione sugli strumenti ad esso concessi per influire sulla decisione del giudice.

Al riguardo, l'art. 299 c.p.p. chiarisce che la persona offesa, o il suo difensore, può presentare al giudicante delle memorie ex art. 121 c.p.p. ${ }^{44}$.

42 Cfr. CASSIBBA, Fabio. Archiviazione e nuovi diritti della persona offesa, in GIULIANI, Livia; ORLANDI, Renzo (a cura di), Indagini preliminari e giudizio di primo grado. Torino: Giappichelli, 2018, p. 127; GIARDA, Angelo. Le nuove indagini preliminari: rinforzo di garanzie ed accelerazioni funzionali, Diritto penale e processo. Milano, n. 10, p. 1305, 2017; GIULIANI, Livia. Indagini preliminari e udienza preliminare, in BARGIS, Marta, Compendio di procedura penale. X ed. Padova: CEDAM, 2020, p. 489.

43 Cfr. SPAGNOLO, Paola. Vittima di reato e diritto all'informazione: un binomio insoddisfacente, Cassazione penale. Milano, n. 10, p. 3485, 2017. In altra prospettiva si è, inoltre, segnalato che l'articolo in parola non implica «comunque il dovere del pubblico ministero di fornire alla persona offesa le informazioni richieste»: così CASSIBBA, Fabio. Archiviazione e nuovi diritti della persona offesa, cit. p. 128.

In argomento v., anche, PARLATO, Lucia. Per la persona offesa ancora piccoli passi verso una più consapevole partecipazione al procedimento. In AA. VV., Le recenti riforme in materia penale. Milano: Wolters Kluwer, 2017, p. 139-167.

44 L'art. 121 c.p.p., cui l'art. 299 c.p.p. espressamente rinvia, si occupa, in realtà, delle memorie e delle richieste delle parti, non già di quelle della persona offesa. Segnala tale incoerenza sistematica: BELLUTA, Hervé. Revoca o sostituzione di misura cautelare e limiti al coinvolgimento della vittima, Diritto penale contemporaneo. Milano, 28 novembre 2013. 
Orbene, queste ultime sono, come è noto, atti di parte intesi ad illustrare ragioni, in fatto o in diritto, a suffragio di una tesi o di una affermazione. Si tratta, quindi, di mezzi che veicolano le argomentazioni del soggetto da cui provengono verso un destinatario - nel caso di specie, il giudice - al fine di influire su di una decisione che spetta a quest'ultimo ${ }^{45}$. Pertanto, nel procedimento in esame, è ragionevole attendersi che la persona offesa si attivi con lo strumento de quo al fine di contestare il merito delle ragioni addotte dall'imputato o dal pubblico ministero a sostegno dell'istanza di mutamento in melius del regime cautelare in atto.

Allargando lo sguardo oltre quanto esplicitamente previsto dalla disposizione in commento, si può osservare come la possibilità di presentare delle memorie non costituisca, in realtà, l'unico strumento a disposizione della vittima. Il difensore di quest'ultima può, infatti, fornire il proprio contributo attraverso la presentazione al giudice dei risultati derivanti dall'indagine difensiva. Al riguardo è noto che, benché la 1.7 dicembre 2000, n. 397 - nell'inserire nel corpo del Libro $\mathrm{V}$ del codice il Titolo VI-bis dedicato alle investigazioni difensive - non abbia fatto alcun esplicito riferimento a tale soggetto quale titolare della legittimazione a compiere questo tipo di attività, la maggior parte degli interpreti concorda nell'attribuire tale facoltà anche all'avvocato nominato dall'offeso ${ }^{46}$.

Relativamente alle modalità di ostensione di quanto raccolto con le forme di cui agli artt. 391-bis e ss. c.p.p., l'art. 391-octies, comma 2 c.p.p. prevede, per quel che qui interessa ${ }^{47}$, la possibilità di presentare,

45 Al riguardo, in generale, ORLANDI, Renzo. L'attività argomentativa delle parti nel dibattimento penale, in AA. VV., La prova nel dibattimento penale. IV ed. Torino: Giappichelli, 2010, p. 3 s. Nonché, con specifico riguardo alle memorie presentate dall'offeso ex art. 299 c.p.p., ZACCHÈ, Francesco. Le cautele fra prerogative dell'imputato e tutela della vittima di reati violenti, cit., p. 676.

GRIFANTINI, Fabio Maria. La persona offesa del reato nella fase delle indagini preliminari. Napoli: Editoriale scientifica, 2012, p. 302; LORENZETTO, Elisa. Il diritto di difendersi indagando nel sistema processuale penale, cit., p. 128 s.; PARLATO, Lucia. Il contributo della vittima tra azione e prova. Palermo: Torri del Vento, 2012, p. 138; TRIGGIANI, Nicola. Le investigazioni difensive. Milano: Giuffrè, 2002, p. 91 s.

47 La disposizione prevede, oltre a quella indicata nel testo, altre due ipotesi di ostensione dei risultati ottenuti dall'attività di investigazione difensiva: la 
nel corso delle indagini preliminari, gli elementi acquisiti direttamente al giudicante affinché questi ne tenga conto nel caso in cui debba adottare una decisione con o senza l'intervento della parte assistita, e quindi anche in vista della sostituzione o della revoca di una misura de libertate ${ }^{48}$.

Sul punto occorre soffermarsi ulteriormente al fine di sgomberare il campo da una possibile obiezione. Giacché, come si è testé osservato, la disposizione da ultimo citata si riferisce espressamente alla «parte assistita», si potrebbe ritenere, facendo perno sulla circostanza che la vittima non può essere considerata una parte in senso tecnico-giuridico, che il patrono della persona offesa, pur potendo svolgere l'attività di ricerca di elementi a favore del proprio assistito, non possa presentarli al giudice per le indagini preliminari nel modo sopra descritto. Un simile ragionamento, però, non meriterebbe di essere condiviso: come si è convincentemente sostenuto in dottrina, la locuzione di cui si discute non deve essere intesa nella sua accezione tecnica, come si desume dal fatto che l'orizzonte temporale in cui si colloca l'art. 391-octies, comma 2 c.p.p. è rappresentato dalle indagini preliminari, segmento procedimentale nel quale agiscono, più propriamente, soggetti e non "parti” ${ }^{49}$.

Deve essere, infine, escluso che l'offeso possa presentare il proprio contributo attraverso dichiarazioni rese oralmente dinanzi al magistrato investito della decisione. Al riguardo va, infatti, osservato che mentre alla persona sottoposta alle indagini è riconosciuto - alle condizioni previste dall'art. 299, comma 3-ter c.p.p. ${ }^{50}$ - il diritto di essere interrogata

presentazione al giudice nel corso dell'udienza preliminare, ovvero il deposito, durante la fase investigativa, nella segreteria del pubblico ministero.

48 Analogamente PAULESU, Pier Paolo, Vittima del reato e processo penale: uno sguardo d'insieme (informazioni, diritti, tutele), cit., p. 152, per il quale «la facoltà della vittima di allegare memorie (art. 121 c.p.p.) in sede di revoca 0 sostituzione delle misure cautelari sottende una pregressa ed autonoma attività di ricerca di dati utili».

${ }^{49}$ Così GRIFANTINI, Fabio Maria. La persona offesa del reato nella fase delle indagini preliminari, cit., p. 300.

${ }^{50}$ L'articolo citato nel testo prevede che il giudice, valutati gli elementi addotti per la revoca o la sostituzione delle misure, prima di procedere possa assumere l'interrogatorio della persona sottoposta alle indagini; facoltà che diventa un obbligo per lo stesso giudicante allorché l'istanza di modifica del regime cautelare sia fondata su elementi nuovi o diversi rispetto a quelli già valutati. Sul punto, anche per gli opportuni riferimenti bibliografici, 
dal giudicante, nessuna disposizione accorda alla vittima del reato all'interno della cornice della fase investigativa - analoga prerogativa. Né è possibile che il giudice per le indagini preliminari proceda sua sponte a sentire l'offeso, dal momento che i suoi poteri probatori officiosi devono intendersi limitati, ai sensi dell'art. 299, comma 4-ter c.p.p., al compimento di accertamenti sulle condizioni di salute o su altre condizioni o qualità personali dell'imputato ${ }^{51}$.

\section{Presidi sanzionatori.}

L'effettività delle prerogative difensive concesse alla persona offesa va ora misurata sul metro della reazione approntata dall'ordinamento in caso di loro indebita pretermissione. In questo senso, l'indagine deve abbracciare tanto l'ipotesi in cui l'illegittima omissione della notificazione dell'istanza ex art. 299 c.p.p. abbia impedito alla vittima di fornire il proprio contributo, tanto il caso in cui - pur correttamente instaurato il contraddittorio - il giudice non abbia tenuto conto delle deduzioni o delle argomentazioni provenienti dall'offeso.

In merito alla prima eventualità, il legislatore è intervenuto in modo espresso, comminando l'inammissibilità dell'istanza. Prima facie, sembrerebbe, quindi, che l'ordinamento si sia dotato di un efficace strumento reattivo quantomeno contro la più macroscopica violazione delle prerogative della vittima in subiecta materia: l'impressione viene, tuttavia, smentita sol che si consideri il caso in cui il giudice, anziché procedere alla declaratoria di inammissibilità, erroneamente si pronunci sulla fondatezza della richiesta indebitamente non notificata alla persona offesa. Occorre, nello specifico, domandarsi se l'originario vizio dell'atto di parte possa estendersi anche al successivo provvedimento giurisdizionale, ovvero se esso vada incontro a qualche forma implicita di sanatoria.

v. BUZZELLI, Silvia. Commento all'art. 299 c.p.p., in ILLUMINATI, Giulio; GIULIANI, Livia (a cura di), Commentario breve al codice di procedura penale. III ed. Padova: CEDAM, 2020, p. 1358 s.

51 Cfr. FIORIO, Carlo. Libertà personale e diritto alla salute. Padova: CEDAM, 2002, p. 283 s.; SPANGHER, Giorgio. La pratica del processo penale. vol. III. Padova: CEDAM, 2014, p. 810. 
Il quesito non si presta ad una pronta ed agevole soluzione, come testimoniato dalla pluralità di soluzioni offerte dagli interpreti. Secondo un primo orientamento, nel caso in esame, l'ordinanza ex art. 299 c.p.p. dovrebbe considerarsi affetta da nullità ai sensi dell'art. 178, lett. c c.p.p., per violazione di disposizioni concernenti l'intervento, l'assistenza e la rappresentanza delle parti private, e come tale rilevabile d'ufficio in ogni stato e grado del giudizio cautelare ${ }^{52}$. Si tratta, però, di una tesi non condivisibile in quanto trascura che, de iure condito, alla persona offesa non è formalmente attribuita la qualità di parte, con conseguente impossibilità di invocare ai fini di cui si discute l'art. 178, lett. c c.p.p.

Riguardata la questione da un differente angolo di visuale, altri sostengono che si dovrebbe, invece, fare ricorso alla categoria dell'invalidità derivata ${ }^{53}$. In questa prospettiva, l'inammissibilità dell'istanza indebitamente non notificata si comunicherebbe all'atto successivo che dalla domanda stessa dipende, ossia all'ordinanza pronunciata ex art. 299 c.p.p. ${ }^{54}$. Orbene, anche questo secondo orientamento non risulta del tutto condivisibile. Poiché, come ricordato in dottrina, l'inammissibilità costituisce un vizio riferibile esclusivamente agli atti di parte di natura

52 Cass. Pen., sez. VI, 22 marzo 2019, n. 27601, Pascale, Rv. 276077; Così Cass. Pen., sez. V, 12 giugno 2017, n. 43103, Urso, Rv. 271009.

53 Per invalidità derivata deve intendersi quel fenomeno di comunicazione di un medesimo vizio da un atto ad uno consecutivo che dal primo dipende dal punto di vista logico giuridico. Al riguardo, FONTI, Rossella. L'inammissibilità degli atti processuali penali. Padova: CEDAM, 2008, p. $42 \mathrm{s.}$

54 In tal senso, ex pluribus, Cass. Pen., sez. VI, 14 novembre 2017, n. 8691, Diritto \& giustizia. Milano, 23 febbraio 2018; Cass. Pen., sez. II, 14 luglio 2016, n. 35576, Fassih, Rv. 267500; Cass. Pen., sez. VI, 23 luglio 2015, n. 35613, p.o. in proc. T., Rv. 264342; Cass. Pen., sez. VI, 5 febbraio 2015, n. 6717, p.o. in proc. D., Giurisprudenza italiana. Milano, n. 3, c. 727, 2015.

Una parte della dottrina che aderisce all'orientamento in parola ritiene, altresì, che nelle ipotesi di cui all'art. 299, comma 3 c.p.p. - in cui, come è noto, il giudice può procedere ex officio ad adeguare, in bonam partem, il regime cautelare in atto - l'ordinanza di sostituzione o revoca pronunciata a seguito della presentazione di un'istanza inammissibile dovrebbe considerarsi del tutto legittima, dal momento che l'esistenza di un potere ufficioso spezzerebbe il legame di interdipendenza logico-giuridica fra domanda di parte e provvedimento decisorio. In tal senso, VALENTINI, Elena. Il ruolo della persona offesa nella procedura di revoca e sostituzione delle misure cautelari personali, cit., p. 239. 
petitoria, il cui riscontro nel caso concreto determina l'inattitudine della richiesta «a vincolare il giudice ad emettere una pronuncia sul merito di essa ${ }^{55}$, appare, invero, assai disagevole sostenere che l'invalidità in parola possa trasmettersi al provvedimento del giudicante ${ }^{56}$.

A tal punto, non resta che prendere atto dell'esistenza, in merito alla corretta soluzione da dare al quesito che ci occupa, di un ventaglio di opzioni interpretative, nessuna delle quali si rivela, però, appagante. Tale circostanza, dovuta principalmente alla scarsa attenzione prestata dal legislatore al tema, rischia di riflettersi negativamente sui diritti partecipativi della vittima in seno al procedimento di cui all'art. 299 c.p.p., nella misura in cui impedisce di dare una risposta sanzionatoria certa alla loro indebita pretermissione.

Non meno problematica si presenta anche la seconda delle ipotesi in esame, relativa alla mancata valutazione da parte del giudice del contributo argomentativo o probatorio dell'offeso. In questo caso, infatti, l'assenza di alcuna comminatoria espressa di nullità impedisce, mercé il principio di tassatività ex art. 177 c.p.p., di ritenere invalida l'ordinanza giudiziale. $\mathrm{Al}$ riguardo va, però, detto che in simili evenienze il provvedimento del giudice, nella misura in cui vanifica la possibilità concessa alla persona offesa di influire sulla decisione de libertate, potrebbe considerarsi affetto da vizio di motivazione ${ }^{57}$, che è possibile - se del caso - far valere attraverso l'attivazione dell'appropriato strumento di impugnazione ${ }^{58}$.

55 Così DELOGU, Tullio. Contributo alla teoria dell'inammissibilità nel diritto processuale penale. Milano: Giuffrè, 1938, p. 51.

56 In dottrina, infatti, la tesi maggioritaria nega che si possa parlare di inammissibilità derivata in capo all'atto del giudice: in tal senso, per tutti, CAPONE, Arturo. L'invalidità nel processo penale. Tra teoria e dogmatica. Padova: CEDAM, 2012, p. 119.

57 Analogamente, in generale, RIVELLO, Pier Paolo. La struttura, la documentazione e la traduzione degli atti, in UBERTIS, Giulio; VOENA, Giovanni Paolo (diretto da), Trattato di procedura penale. vol. X.1. Milano: Giuffrè, 1999, p. 104.

Secondo alcuni interpreti, invece, il giudice non sarebbe tenuto a tenere in considerazione le ragioni in fatto e in diritto introdotte dalla persona offesa: in tal senso QUATTROCOLO, Serena. Vittima e processo penale: commistioni di ruoli e di funzioni, cit., p. 583; ZACCHÈ, Francesco. Le cautele fra prerogative dell'imputato e tutela della vittima di reati violenti, cit., p. 676.

V., sul punto, infra, $\S 5$. 


\section{Modalità di CONTESTAZIONE DELLA VIOLAZIONE DELLE PREROGATIVE PARTECIPATIVE DELL'OFFESO EX ART. 299 C.P.P.}

Se le considerazioni poc'anzi formulate lasciano insoddisfatti circa la capacità del sistema di reagire adeguatamente alla violazione delle garanzie partecipative della persona offesa all'interno del procedimento di revoca o sostituzione di una misura cautelare, la sensazione risulta addirittura rafforzata sol che si volga l'attenzione verso il profilo relativo alle modalità con le quali contestare il provvedimento adottato senza tener conto delle osservazioni della vittima, ovvero senza che quest'ultima sia stata messa nelle condizioni di fornire il proprio contributo ${ }^{59}$.

Al riguardo, va osservato come già i primi commentatori del D.L. 14 agosto 2013, n. 93, convertito dalla 1.15 ottobre 2013, n. 119 abbiano indicato l'aspetto in esame quale punto debole del nuovo istituto, tale da incrinarne l'effettività ${ }^{60}$. Il legislatore non ha, infatti, espressamente conferito alla vittima la legittimazione ad impugnare il provvedimento adottato in spregio dei suoi diritti informativi e di partecipazione di cui all'articolo 299 c.p.p. Si è, pertanto, correttamente sostenuto, facendo perno sul principio di tassatività dei mezzi di impugnazione, che nel caso che ci occupa l'unico strumento a disposizione della persona offesa è costituito dall'art. 572 c.p.p., in base al quale essa può solamente sollecitare il pubblico ministero ad introdurre il mezzo di impugnazione adeguato,

59 Vale la pena ricordare come il tema della legittimazione della persona offesa ad impugnare le decisioni che dispongono la cessazione o la modulazione in melius del regime cautelare applicato all'imputato sia affrontato solo tangenzialmente dalle fonti europee. In questo senso, il Punto n. 33 del Considerando della Direttiva 2012/29/UE dispone che le vittime dovrebbero essere informate in merito all'eventuale diritto di presentare ricorso avverso una decisione di scarcerazione dell'autore del reato, se tale diritto esiste nell'ordinamento nazionale.

Orbene, la collocazione della disposizione in commento e la sua formulazione letterale non consentono di ritenere che l'atto normativo in parola abbia introdotto un obbligo per gli Stati membri di garantire all'offeso la possibilità di impugnare i provvedimenti in materia de libertate.

60 DIDDI, Alessandro. Chiaroscuri nella nuova disciplina sulla violenza di genere, cit., p. 100. 
ovverosia l'appello di cui all'art. 310 c.p.p..$^{61}$. Tale congegno, però, è parso per vero, non da oggi - del tutto inefficace a tutelare le ragioni del soggetto che ci occupa. Come sottolineato in dottrina, esso finisce per rimettere la tutela delle prerogative dell'offeso dal reato alla discrezionalità dell'organo inquirente, cui viene posto, nel caso in cui non accolga la sollecitazione ad impugnandum, il solo obbligo di motivare il proprio diniego ${ }^{62}$.

Proprio per superare i limiti ora segnalati, la giurisprudenza ha percorso strade alternative, che - benché non supportate dal dato normativo, e dunque non condivisibili sul piano metodologico, ancor prima che per i risultati raggiunti - paiono di estremo interesse per l'interprete, in quanto espressione di quella tendenza giurisprudenziale a farsi carico delle esigenze di tutela della persona offesa dal reato e, a tal fine, a ricercare, anche al prezzo di forzature delle consolidate categorie codicistiche, nuove soluzioni. Al riguardo, mette conto di segnalare come, accanto ad alcune pronunce che hanno fatto applicazione dell'art. 572 c.p.p. nel senso sopra indicato ${ }^{63}$, in seno alla Suprema Corte si siano formati altri due orientamenti ${ }^{64}$.

61 Sull'appello cautelare ex art. 310 c.p.p. v, da ultimo, MAGGIO, Paola. Le impugnazioni delle misure cautelari personali, in UBERTIS, Giulio; VOENA, Giovanni Paolo (diretto da), Trattato di procedura penale. vol. XX. Milano: Giuffrè, 2018, p. 405 s.

In questo senso, CAMPOLI, Enrico. La tutela della persona offesa nella violenza di genere: brevi riflessioni sulle novelle processuali, Archivio della nuova procedura penale. Piacenza, n. 3, p. 222, 2014, il quale sostiene che lo strumento di cui all'art. 572 c.p.p. «appare non solo del tutto blando, ma anche, in alcuni casi, potenzialmente in "conflitto di interessi" con le posizioni che l'organo dell'accusa può aver assunto in quello specifico procedimento».

Alla segnalazione della lacuna è, peraltro, seguita, da parte di taluni interpreti, una sollecitazione a valutare l'opportunità di dare maggior spazio alla persona offesa nei giudizi sulle impugnazioni de libertate: v., in tal senso, MARZADURI, Enrico. Diritto di difesa e tempi del procedimento dinanzi al Tribunale della libertà, in GIULIANI, Livia (a cura di), La riforma delle misure cautelari personali. Torino: Giappichelli, 2015, p. 225.

63 In tal senso, Cass. Pen., sez. V, 17 ottobre 2017, n. 5820, p.o. in proc. G., Diritto penale e processo, Milano, n. 2, p. 244 ss., 2019; Cass. Pen., sez. V, 17 maggio 2017, n. 54319, p.o. in proc. B. e a., Rv. 272005.

64 Gli orientamenti cui ci si riferisce nel testo riguardano vicende in cui alla persona offesa non veniva notificata l'istanza di sostituzione o revoca di una misura cautelare coercitiva, in violazione dell'art. 299 c.p.p. Stando ai 
Orbene, un primo schema di pensiero ritiene, andando ben oltre il dato letterale, che alla vittima del reato debba riconoscersi la possibilità di far valere la mancata notificazione della richiesta di revoca o sostituzione delle cautele attraverso la presentazione diretta dell'appello de libertate di cui all'art. 310 c.p.p..$^{65}$, senza, quindi, che si renda necessaria la mediazione del pubblico ministero. Si tratta, come anticipato, di un'impostazione non convincente. Dal momento che l'art. 310, comma 1 c.p.p. attribuisce expressis verbis la legittimazione ad azionare il rimedio ivi contemplato al pubblico ministero, all'imputato e al suo difensore, la soluzione testé ricordata si pone in patente contrasto con il canone scolpito nell'art. 568, comma 3 c.p.p., secondo cui il diritto di impugnazione spetta soltanto a colui al quale la legge espressamente lo conferisce.

Al fine di superare l'ostacolo rappresentato dall'art. 568, comma 3 c.p.p. altro e diverso indirizzo interpretativo ha ritenuto possibile fare applicazione dell'art. 568, comma 2 c.p.p., a norma del quale sono sempre soggetti a ricorso per cassazione, quando non altrimenti impugnabili, i provvedimenti con i quali il giudice decida sulla libertà personale. In questa prospettiva, si è affermato che, in virtù di un'interpretazione conforme alle fonti sovranazionali che si occupano dei diritti della persona offesa dal reato, l'esplicita previsione della sanzione di inammissibilità «comporta conseguentemente la possibilità di farla valere dalla parte nei cui confronti la sanzione è stata eminentemente apprestata ${ }^{66}$. Cosicché, a fronte del silenzio del legislatore circa il rimedio a disposizione dell'offeso, spetterebbe all'interprete ritrovare all'interno dell'ordinamento il mezzo

provvedimenti editi, non risulta che la giurisprudenza si sia mai espressa in relazione al diverso caso in cui il giudice, pur avendo contezza del contributo della vittima, abbia disposto il mutamento del regime de libertate senza tenerne conto: anche in questa eventualità, deve ritenersi che l'unico strumento a disposizione dell'offeso sia la formulazione di una sollecitazione, ex art. 572 c.p.p., al pubblico ministero perché impugni il provvedimento attraverso il mezzo dell'appello di cui all'art. 310 c.p.p.

65 Tale interpretazione viene sostenuta in Cass. Pen., sez. I, 28 giugno 2016, n. 51402, Zacheo, Diritto \& Giustizia. Milano, n. 95, p. 2, 2016; Cass. Pen., sez. VI, 23 luglio 2015, n. 35613, p.o. in proc. T., Rv. 264342; Cass. Pen., sez. V, 31 marzo 2015, n. 35735, p.o. in proc. S., Rv. 265866.

66 Così, Cass. Pen., sez. V, 20 settembre 2016, n. 7404, p.o. in proc. M., Cassazione penale. Milano, n. 12, p. 4428, 2017. 
più appropriato, tenendo in adeguata considerazione i principi che reggono la disciplina delle impugnazioni. Poiché fra questi ultimi si rinviene, come ricordato, il principio di tassatività, l'unica soluzione praticabile consisterebbe nel riconoscere la legittimazione della vittima a proporre ricorso per cassazione, ai sensi dell'art. 568, comma 2 c.p.p. Tale, ultimo strumento, infatti, trovando un esplicito referente costituzionale nell'art. 111, comma 7 Cost. - a mente del quale è sempre ammesso ricorso in cassazione per violazione di legge contro i provvedimenti sulla libertà personale - potrebbe essere correttamente invocato anche dalla persona offesa, pure in assenza di una esplicita previsione in tal senso ${ }^{67}$.

Anche questo secondo orientamento prova troppo. A non convincere è, in particolare, il richiamo alla garanzia di cui all'art. 111, comma 7 Cost. quale fondamento del diritto della persona offesa ad impugnare i provvedimenti in materia de libertate, giacché essa, più propriamente, mira a offrire uno strumento di tutela al soggetto che soffra la limitazione della propria libertà personale ${ }^{68}$ (ovvero, secondo parte della dottrina, anche alla parte pubblica chiamata a salvaguardare le istanze di sicurezza della comunità ${ }^{69}$.

67 Tale soluzione ha trovato accoglimento in Cass. Pen., sez. V, 20 settembre 2016, n. 7404, p.o. in proc. M., cit., p. 4426 s.; Cass. Pen., sez. VI, 9 febbraio 2016, n. 6864, p.o. in proc. P., Cassazione penale. Milano, n. 10, p. 3753, 2016; Cass. Pen., sez. VI, 5 febbraio 2015, n. 6717, p.o. in proc. D., cit.

Per una rassegna degli orientamenti ricordati v. ROMANELLI, Bartolomeo. Omessa notifica alla persona offesa della richiesta di revoca o sostituzione di misura cautelare coercitiva: problemi definitori e rimedi in sede di impugnazione, Cassazione penale. Milano, n. 12, p. 4429-4439, 2017.

Al riguardo, GREVI, Vittorio. Libertà personale dell'imputato e Costituzione. Milano: Giuffrè, 1976, p. 234, per il quale la funzione primaria dell'art. 111, comma 7 Cost. consisterebbe nel «sancire la necessità che in ordine a tutti i provvedimenti restrittivi della libertà personale dell'imputato venga quantomeno assicurata, accanto alle garanzie della riserva di legge e della riserva di giurisdizione fissate dall'art. 13 Cost., la garanzia del successivo controllo di legittimità in cassazione».

69 In proposito, va detto che parte della dottrina, facendo leva sul riferimento - contenuto nell'art. 111 , comma 7 Cost. - ai provvedimenti sulla libertà personale, non già a quelli limitativi di quest'ultima, ha sottolineato come la disposizione de qua parrebbe fornire copertura alla legittimazione al ricorso anche da parte di soggetti diversi dalla persona ristretta (segnatamente della 


\section{ConCLUSIONi}

Tirando le fila del discorso sin qui condotto, pare giunto il momento per formulare alcune riflessioni conclusive. L'analisi circa la consistenza delle facoltà riconosciute alla persona offesa nel procedimento di sostituzione o revoca delle misure ex art. 299, comma 3 c.p.p. ha evidenziato l'inadeguatezza dell'attuale disciplina a garantirle un vero e proprio diritto al contraddittorio cautelare.

In questa prospettiva si è osservato come, nel momento della presentazione di una istanza ex art. 299 c.p.p., l'orizzonte conoscitivo della vittima circa gli atti a disposizione per la decisione sia assai limitato, specialmente se paragonato a quello degli altri contendenti processuali. Un deficit cognitivo, quest'ultimo, che depotenzia grandemente l'efficacia dell'intervento del soggetto in parola.

Una più significativa considerazione per la posizione dell'offeso si registra, invece, spostando l'attenzione sul compendio di strumenti che esso può utilizzare per influire sulla decisione del giudice, che si compone - in questo caso, su di un piano di tendenziale parità rispetto all'accusato - di atti a contenuto tanto argomentativo (memorie), quanto probatorio (risultati delle investigazioni difensive).

Si è visto come al riconoscimento formale di diritti partecipativi alla vittima nel procedimento de libertate non si accompagni, però, un adeguato apparato di sanzioni e rimedi, per i casi di disapplicazione nel caso concreto delle prerogative riconosciute all'offeso. I dubbi che circondano tanto la natura dell'invalidità derivante dall'indebita mancata attivazione dell'interpello cautelare ex art. 299, comma 3 c.p.p., tanto le modalità con cui far valere tale vizio, lasciano, invero, insoddisfatti circa la capacità dell'ordinamento di garantire, in concreto, le prerogative in parola che, in astratto, trovano invece una perentoria

parte pubblica). In tal senso CHIAVARIO, Mario. Processo penale e garanzie della persona. III ed. Milano: Giuffrè, 1984, p. 257.

Pur intesa in questo senso, la disposizione in commento non sembra utilmente invocabile al fine di enucleare il diritto della persona offesa a ricorrere per cassazione in materia de libertate, dal momento che una simile interpretazione finirebbe per travolgere la ratio di garanzia della norma. 
affermazione ${ }^{70}$. Tanto da condurre la giurisprudenza a farsi carico di colmare le lacune lasciate dal legislatore attraverso interpretazioni creative che, come si è visto, benché ispirate a comprensibili logiche di giustizia sostanziale, risultano incompatibili con il doveroso rispetto del principio di legalità in materia de libertate.

Alla luce delle considerazioni poc'anzi formulate, sembra che la risposta all'interrogativo posto in premessa debba essere negativa: l'interpello cautelare con l'offeso di cui all'art. 299 c.p.p. si dimostra, infatti, non all'altezza delle aspettative che hanno accompagnato la sua introduzione. Si avverte, in particolare, la sensazione di trovarsi ancora ben lontani dal riconoscimento alla persona offesa di un vero e proprio diritto a partecipare attivamente alla cognizione de libertate.

\section{Bibliografia}

AMODIO, Ennio. Relazione introduttiva. In AA. VV. I nuovi orizzonti della giustizia penale europea. Milano: Giuffrè, 2015, p. 15-27.

BELLUTA, Hervé. Quale ruolo per la vittima nel processo penale italiano? Revista Brasileira De Direito Processual Penal, Porto Alegre, vol. 5, n. 1, p. 73-92, 2019.

BELLUTA, Hervé. Revoca o sostituzione di misura cautelare e limiti al coinvolgimento della vittima. Diritto penale contemporaneo, Milano, 28 novembre 2013.

BONINI, Valentina. Il sistema di protezione della vittima e i suoi riflessi sulla libertà personale, Padova: CEDAM, 2018.

BONTEMPELLI, Manfredi. Novità nelle procedure di revoca e sostituzione. In: DIDDI, Alessandro; GERACI, Rosa Maria (a cura di). Misure cautelari ad personam in un triennio di riforme. Torino: Giappichelli, 2015, p. 143-167.

BRONZO, Pasquale. Le "nuove" misure prescrittive. In AA. VV. Il pluralismo delle misure cautelari personali. Padova: CEDAM, 2017, p. 55-95.

BUZZELLI, Silvia. Commento all'art. 299 c.p.p. In ILLUMINATI, Giulio; GIULIANI, Livia (a cura di). Commentario breve al codice di procedura penale. III ed. Padova: CEDAM, 2020, p. 1352-1364.

${ }^{70}$ Cfr. QUATTROCOLO, Serena. Vittima e processo penale: commistioni di ruoli e di funzioni, cit., p. 583. 
CANZIO, Giovanni. La tutela della vittima nel sistema delle garanzie processuali: le misure cautelari e la testimonianza "vulnerabile". Diritto penale e processo, Milano, n. 8, p. 985-992, 2010.

CAPONE, Arturo. L'invalidità nel processo penale. Tra teoria e dogmatica. Padova: CEDAM, 2012.

CAMPOLI, Enrico. La tutela della persona offesa nella violenza di genere: brevi riflessioni sulle novelle processuali. Archivio della nuova procedura penale, Piacenza, n. 3, p. 221-224, 2014.

CARACENI, Lina. Misure cautelari pro victima e diritti di libertà dell'accusato: a proposito di una convivenza faticosa. Archivio della nuova procedura penale, Piacenza, n. 3, p. 254-270, 2017.

CASSIBBA, Fabio. Archiviazione e nuovi diritti della persona offesa. In GIULIANI, Livia; ORLANDI, Renzo (a cura di). Indagini preliminari e giudizio di primo grado. Torino: Giappichelli, 2018, p. 119-158.

CATALANO, Elena Maria. La tutela della vittima nella direttiva 2012/29/UE e nella giurisprudenza delle corti europee. Rivista italiana di diritto e procedura penale, Milano, n. 4, p. 1789-1814, 2014.

CHIAVARIO, Mario. Processo penale e garanzie della persona. III ed. Milano: Giuffrè, 1984.

CRISTIANI, Antonio. Aspetti problematici del contraddittorio nel riesame dei provvedimenti restrittivi della libertà personale. Rivista italiana di diritto e procedura penale. Milano, n. 3, p. 962-982, 1984.

DE AMICIS, Gaetano. Appunti per una ricostruzione sistematica del diritto alla consultazione degli atti processuali. Cassazione penale, Milano, n. 3, p. 978-984, 1996.

DELOGU, Tullio. Contributo alla teoria dell'inammissibilità nel diritto processuale penale. Milano: Giuffrè, 1938.

DI CHIARA, Giuseppe. Linee di sistema della funzione giudiziale preliminare. Rivista di diritto processuale, Milano, n. 1, p. 229-259, 2003.

DI CHIARA, Giuseppe. voce Incidente probatorio. In Enciclopedia del diritto. Agg. VI. Milano: Giuffrè, 2002, p. 546-565.

DIDDI, Alessandro. Chiaroscuri nella nuova disciplina sulla violenza di genere. Processo penale e giustizia, Torino, n. 2, p. 91-111, 2014. 
FERRAIOLI, Marzia. Il ruolo di «garante» del giudice per le indagini preliminari. IV ed. Padova: CEDAM, 2014.

FIORIO, Carlo. Libertà personale e diritto alla salute. Padova: CEDAM, 2002.

FONTI, Rossella. L'inammissibilità degli atti processuali penali. Padova: CEDAM, 2008.

GIARDA, Angelo. Le nuove indagini preliminari: rinforzo di garanzie ed accelerazioni funzionali. Diritto penale e processo, Milano, n. 10, p. 1302-1306, 2017.

GIOSTRA, Glauco. voce Contraddittorio (principio del) II) Diritto processuale penale. In Enciclopedia giuridica Treccani. vol. VIII. Roma: Treccani, 2001, p. 1-13.

GIULIANI, Livia. Indagini preliminari e udienza preliminare. In BARGIS, Marta, Compendio di procedura penale. X ed. Padova: CEDAM, 2020, p. 418-564.

GIULIANI, Livia. Autodifesa e difesa tecnica nei procedimenti de libertate. Padova: CEDAM, 2012.

GIULIANI, Livia. Rimessione del processo e valori costituzionali. Torino: Giappichelli, 2002.

GREVI, Vittorio. Alla ricerca di un processo penale «giusto». Milano: Giuffrè, 2000.

GREVI, Vittorio. Libertà personale dell'imputato e Costituzione. Milano: Giuffrè, 1976.

GRIFANTINI, Fabio Maria. La persona offesa del reato nella fase delle indagini preliminari. Napoli: Editoriale scientifica, 2012.

LEONASI, Raffaele; FERRANTI, Donatella. Il contraddittorio nell'applicazione, revoca e modifica delle misure cautelari. Cassazione penale. Milano, n. 8-9, p. 1651-1654, 1990.

LOMBARDI, Filippo. Tutela della vittima nella vicenda cautelare e obbligo di notificare l'istanza di revoca (o modifica) della misura. Processo penale e giustizia. Milano, n. 6, p. 1517-1532, 2020.

LORENZETTO, Elisa. Il diritto di difendersi indagando nel sistema processuale penale. Napoli: ESI, 2013.

MAGGIO, Paola. Le impugnazioni delle misure cautelari personali. In UBERTIS, Giulio; VOENA, Giovanni Paolo (diretto da). Trattato di procedura penale. vol. XX. Milano: Giuffrè, 2018.

MANZIONE, Domenico. Commento all'art. 299 c.p.p. In CHIAVARIO, Mario (a cura di). Commento al codice di procedura penale. Agg. III. Torino: UTET, 1998, p. 301-312. 
MARCHETTI, Filippo. L'intervento dell'offeso nel procedimento di revoca e sostituzione di una misura cautelare personale nuovamente sotto la lente della Suprema Corte. Diritto penale contemporaneo, Milano, n. 6, p. 121-133, 2019.

MARZADURI, Enrico. Diritto di difesa e tempi del procedimento dinanzi al Tribunale della libertà. In GIULIANI, Livia (a cura di). La riforma delle misure cautelari personali. Torino: Giappichelli, 2015, p. 211-248.

MARZADURI, Enrico. voce Misure cautelari personali (principi generali e disciplina). In Digesto delle discipline penalistiche. Vol. VIII. Torino: UTET, 1994, p. 59-98.

MOLARI, Alfredo. Introduzione. In AA. VV. Il contraddittorio tra Costituzione e legge ordinaria. Milano: Giuffrè, 2002, p. 137-145.

NAPPI, Aniello. Guida al codice di procedura penale. X ed. Milano: Giuffrè, 2007.

NEGRI, Daniele. Le misure cautelari a tutela della vittima: dietro il paradigma flessibile, il rischio di un'incontrollata prevenzione. Giurisprudenza italiana, Milano, n. 2, c. 467-474, 2012.

ORLANDI, Renzo. L'attività argomentativa delle parti nel dibattimento penale. In AA. VV., La prova nel dibattimento penale. IV ed. Torino: Giappichelli, 2010, p. 3-66.

PANSINI, Carla. Contributo dell'offeso e snodi procedimentali. Padova: CEDAM, 2004.

PARLATO, Lucia. Per la persona offesa ancora piccoli passi verso una più consapevole partecipazione al procedimento. In AA. VV., Le recenti riforme in materia penale. Milano: Wolters Kluwer, 2017, p. 139-167.

PARLATO, Lucia. Il contributo della vittima tra azione e prova. Palermo: Torri del Vento, 2012.

PAULESU, Pier Paolo, Vittima del reato e processo penale: uno sguardo d'insieme (informazioni, diritti, tutele). In BARGIS, Marta; BELLUTA, Hervè (a cura di). Vittime di reato e sistema penale. Torino: Giappichelli, 2017, p. 127-166.

QUAGLIERINI, Corrado, Le parti privati diverse dall'imputato e l'offeso dal reato. In UBERTIS, Giulio; VOENA Giovanni Paolo (diretto da). Trattato di procedura penale. vol. VIII. Milano: Giuffrè, 2003.

QUATTROCOLO, Serena. Vittima e processo penale: commistioni di ruoli e di funzioni. Rivista italiana di medicina legale, Milano, n. 2, p. 577-598, 2018.

QUATTROCOLO, Serena. Vulnerabilità e individual assessment: l'evoluzione dei parametri di identificazione. In BARGIS, Marta; BELLUTA, Hervè (a cura di). Vittime di reato e sistema penale. Torino: Giappichelli, 2017, p. 297-324. 
RIVELLO, Pier Paolo. La struttura, la documentazione e la traduzione degli atti. In UBERTIS, Giulio; VOENA, Giovanni Paolo (diretto da). Trattato di procedura penale. vol. X.1. Milano: Giuffrè, 1999.

ROMANELLI, Bartolomeo. La persona offesa vulnerabile nel procedimento penale. Milano: EDUCatt, 2020.

ROMANELLI, Bartolomeo. Omessa notifica alla persona offesa della richiesta di revoca o sostituzione di misura cautelare coercitiva: problemi definitori e rimedi in sede di impugnazione. Cassazione penale, Milano, n. 12, p. 4429-4439, 2017.

RUGGERI, Stefano. Equality of arms, impartiality of the judiciary and the role of the parties in the pre-trial inquiry: the perspective of Italian criminal justice. Revista Brasileira De Direito Processual Penal, Porto Alegre, vol. 4, n. 2, p. 559-603, 2018.

RUGGIERO, Rosa Anna. La tutela processuale della violenza di genere, Cassazione penale. Milano: Giuffrè, n. 6, p. 2352-2359, 2014.

RUSSO, Carmine. Femminicidio. Milano: Giuffrè, 2013.

SPAGNOLO, Paola. Vittima di reato e diritto all'informazione: un binomio insoddisfacente. Cassazione penale, Milano, n. 10, p. 3482-3490, 2017.

SPANGHER, Giorgio. La pratica del processo penale. vol. III. Padova: CEDAM, 2014.

TODARO, Guido. Il sistema italiano di tutela della vittima: analisi e prospettive. In LUPARIA, Luca (a cura di). Lo statuto europeo delle vittime di reato. Padova: CEDAM, 2015, p. 99-115.

TRIGGIANI, Nicola. Le investigazioni difensive. Milano: Giuffrè, 2002.

UBERTIS, Giulio. Sistema di procedura penale. IV ed. vol. I. Milano: Giuffrè, 2017. VALENTINI, Elena. Il ruolo della persona offesa nella procedura di revoca e sostituzione delle misure cautelari personali. In CURI, Francesca (a cura di). Ordine pubblico e sicurezza nel governo della città. Bologna: BUP, 2016, p. 225-140.

VALENTINI, Elena. La domanda cautelare nel sistema delle cautele personali. Bologna: BUP, 2012.

ZACCHÈ, Francesco. Criterio di necessità e misure cautelari. Milano: Giuffrè, 2018.

ZACCHÈ, Francesco. Le cautele fra prerogative dell'imputato e tutela della vittima di reati violenti. Rivista italiana di diritto e procedura penale, Milano, n. 2, p. 646$677,2015$. 


\section{Additional information and author's declarations (scientific integrity)}

Conflict of interest declaration: the author confirms that there are no conflicts of interest in conducting this research and writing this article.

Declaration of authorship: all and only researchers who comply the authorship requirements of this article are listed as authors; all coauthors are fully responsible for this work in its entirety.

Declaration of originality: the author assures that the text here published has not been previously published in any other resource and that future republication will only take place with the express indication of the reference of this original publication; he also attests that there is no third party plagiarism or self-plagiarism.

\section{Editorial process dates}

(http://www.ibraspp.com.br/revista/index.php/RBDPP/about/editorialPolicies)

- Submission: 13/07/2021

- Desk review and plagiarism check: 02/08/2021

- Review 1: 18/09/2021

- Review 2: 22/09/2021

- Preliminary editorial decision: 22/09/2021

- Correction round return: 01/10/2021

- Final editorial decision: 01/10/2021

\section{Editorial team}

- Editor-in-chief: 1 (VGV)

- Associated-editor: 2 (BC, LG)

- Reviewers: 2 


\section{HOW TO CITE (ABNT BRAZIL):}

MARCHETTI, Filippo. Il diritto di difesa della vittima nel procedimento di revoca o sostituzione delle misure cautelari personali durante la fase delle indagini preliminari. Revista Brasileira de Direito Processual Penal, vol. 7, n. 3, p. 1825-1858, set./dez. 2021. https://doi.org/10.22197/rbdpp.v7i3.630

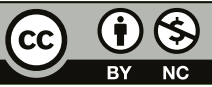

Esta obra está licenciada com uma Licença Creative Commons Atribuição-NãoComercial 4.0 Internacional. 